\title{
Ein korrektes Verhältnis zum Staat
}

\author{
Elisabeth Essbaumer
}

\section{Relevanz}

Ein korrektes Verhältnis zum Staat zeichnet sich durch Gemeinsinn, Ehrlichkeit und gegenseitiges Vertrauen aus. Zivile Tugenden begünstigen nicht nur eine hohe Steuermoral, sondern ermöglichen auch bessere Politiklösungen anderswo. Die Arbeitslosenversicherung wird sehr teuer, wenn ein wachsender Teil der Arbeitslosen lieber großzügiges Arbeitslosengeld empfängt anstatt sich für die Beschäftigung zu rüsten und die Mühen der Jobsuche einzugehen. Dann muss der Staat eher auf rigorosen Kündigungsschutz setzen, um vor dem Arbeitslosenrisiko zu schützen und die Kosten der Versicherung zu begrenzen. Das würde aber die existierende Beschäftigung zementieren und verhindern, dass Arbeit von wenig produktiven Jobs zu den aufstrebenden Branchen fließt, wo das Wachstum und die zukunftsträchtige Beschäftigung stattfinden. Es lohnt sich also, die zivilen Tugenden der Bürger zu pflegen, damit der Staat auf die Arbeitslosensicherung anstatt den Kündigungsschutz setzen und die soziale Sicherheit wachstumsverträglicher gestalten kann.

\section{Quelle}

Algan and Cahuc (2009), Civic Virtue and Labor Market Institutions, American Economic Journal: Macroeconomics, 111-145.

\author{
E. Essbaumer $(\bowtie)$ \\ Universität St.Gallen, St.Gallen, Schweiz \\ E-Mail: elisabeth.essbaumer@unisg.ch
}


Ein Staat entspricht dem Wesen seiner Bürger, kommentiert Platon in der Politeia, einem seiner Hauptwerke über die Staatstheorie. Der Begriff „Bürgerliche Tugend" meint hier weniger die persönliche Sittsamkeit, als vielmehr das Verantwortungsbewusstsein des Einzelnen für die Gemeinschaft und die Bereitschaft, sich für das Allgemeinwohl einzusetzen. Dafür interessieren sich auch heute, mehr als 2000 Jahre später, die beiden Ökonomen Yann Algan und Pierre Cahuc. Sie untersuchen für den Zeitraum zwischen 1980 und 2000, inwiefern sich ein hoher Gemeinsinn auf die Art der Institutionen zum Schutz vor Arbeitslosigkeit in verschiedenen OECD Staaten und einigen postkommunistischen Ländern ausgewirkt hat. Wie kann ein hoher Gemeinsinn darauf Einfluss haben, ob die Länder eher auf die Arbeitslosenversicherung oder einen rigorosen Kündigungsschütz setzen? Die Forscher erheben den Grad an Gemeinsinn aus einer Befragung über die Einstellung gegenüber einem möglichen Ausnutzen des Sozialstaates. Sie messen damit, in wieweit eine ungerechtfertigte Inanspruchnahme von Sozialleistungen eher als Kavaliersdelikt oder als Betrug empfunden wird.

Die grundsätzliche Idee ist, dass es für den Staat umso teurer ist, ein Arbeitslosengeld zu zahlen, je weniger Hemmungen seine Bürger besitzen, ihn auszunutzen. In diesem Fall ist es für den Staat einfacher und kostengünstiger, den Kündigungsschutz auszubauen, um auf diesem Weg die Arbeitnehmer vor der Arbeitslosigkeit zu schützen. Das wäre für den Strukturwandel und für die Umlenkung der Arbeit auf die Wachstumsbranchen negativ. Die Autoren decken einen wesentlichen Zusammenhang auf: Scheuen sich Bürger, den Sozialstaat zu betrügen, dann können die Staaten relativ großzügige Arbeitslosenleistungen ausrichten. Ist der Gemeinsinn nur wenig ausgeprägt, setzen die Staaten eher auf Kündigungsschutz, um den Arbeitgebern Entlassungen zu erschweren und so die Kosten der Arbeitslosenversicherung zu begrenzen. Die empirische Analyse zeigt deutliche Unterschiede zwischen den nordisch-skandinavischen Ländern mit einem besser ausgeprägten Gemeinsinn auf der einen Seite und den kontinentalund südeuropäischen Staaten auf der anderen Seite.

Es ist für den Staat umso teurer, ein Arbeitslosengeld zu zahlen, je eher seine Bürger bereit sind, ihn finanziell auszunutzen.

Die Forscher belegen diesen Zusammenhang mit empirischer Evidenz. Dafür untersuchen sie Daten des World Value Survey (WVS), einer Befragung mit drei Erhebungswellen in den Jahren 1980, 1990 und 2000. Algan und Cahuc werten die Antworten von 76221 Personen aus 20 OECD Staaten sowie aus den drei postkommunistischen Staaten Ungarn, Tschechien und Polen auf folgende Frage aus: „Denken Sie, dass es gerechtfertigt ist, staatliche Leistungen in Anspruch zu nehmen, auf die man keinen Anspruch hat?“ Die größte Ablehnung kommt 
aus Dänemark. Dort antworten durchschnittlich $88 \%$ der Befragten, ein solcher Anspruch sei nie gerechtfertigt. Dahinter folgen weitere nordische Staaten wie Norwegen, Schweden und die Niederlande mit Werten um die $80 \%$. Auf der anderen Seite stehen kontinentaleuropäische und mediterrane Staaten, wo durchschnittlich nur etwa $65 \%$ der Teilnehmer die Frage mit „nie“ beantworten. Bei den 2491 Befragten aus der Schweiz liegt der Wert bei knapp unter $70 \%$ etwas darüber. Den geringsten Anteil an Teilnehmern, die einen nicht gerechtfertigten Anspruch vollständig ablehnen, weist Griechenland mit etwas über $20 \%$ auf.

Nur etwa $20 \%$ der griechischen Befragten sprechen sich gegen das mögliche Hintergehen des Sozialstaates aus. In Dänemark sind es $88 \%$.

Es ist natürlich davon auszugehen, dass neben der Herkunft auch andere Faktoren sich auf die Einstellung einer Person gegenüber dem Sozialstaat auswirken. Deswegen untersuchen die Forscher auch den Einfluss von sozioökonomischen Aspekten, wie beispielsweise das Geschlecht der Befragten, deren Bildungsstand, oder auch die Einkommensklasse. So erhöht beispielsweise ein zusätzliches Jahr an Bildung die Wahrscheinlichkeit, einen Sozialstaatsbetrug abzulehnen, um $1 \%$. Protestantisch zu sein erhöht die Wahrscheinlichkeit um durchschnittlich $3 \%$ im Vergleich zu nicht religiösen Personen. Die Einflussstärke solcher persönlichen Faktoren ist jedoch deutlich geringer als der Einfluss des Herkunftslandes.

Da Dänemark den höchsten Wert an Gemeinsinn erreicht, gemessen an der Häufigkeit der Ablehnung von Sozialbetrug, wird das Land als Referenzpunkt angesehen. In die nachfolgende Analyse geht daher nicht der absolute Wert des Anteils der Antworten ein, sondern nur der Unterschied zwischen Befragten aus anderen Ländern zu denen aus Dänemark. Zum Beispiel ist in Spanien die Wahrscheinlichkeit einer Ablehnung bzw. der Anteil der Antworten, dass man den Bezug von unrechtmäßigen Leistungen ablehnt, um $32 \%$ niedriger als in Dänemark. In Italien ist die Wahrscheinlichkeit um $25 \%$ geringer. Diese Werte sind in Abb. 1 auf der horizontalen Achse abgetragen. Dänemark als Referenzpunkt weist einen Wert von null auf. Die Ergebnisse der Umfrage werden mit einem Indikator für die Ausgestaltung der Arbeitsmarktinstitutionen verglichen. Dieser ist auf der vertikalen Achse abgebildet und zeigt die Zahlungen pro Arbeitslosen im Verhältnis zu einem OECD Indikator für den Beschäftigungsschutz. Ein vergleichsweiser hoher Wert auf dieser Achse sagt aus, dass der Staat einen Schwerpunkt auf Geldzahlungen legt. Bei einem niedrigeren Wert ist der Kündigungsschutz verhältnismäßig stärker ausgebaut.

In Spanien ist die Wahrscheinlichkeit anzugeben, dass man den Bezug von unrechtmäßigen Leistungen ablehnt, im Durchschnitt um $32 \%$ niedriger als in Dänemark. 


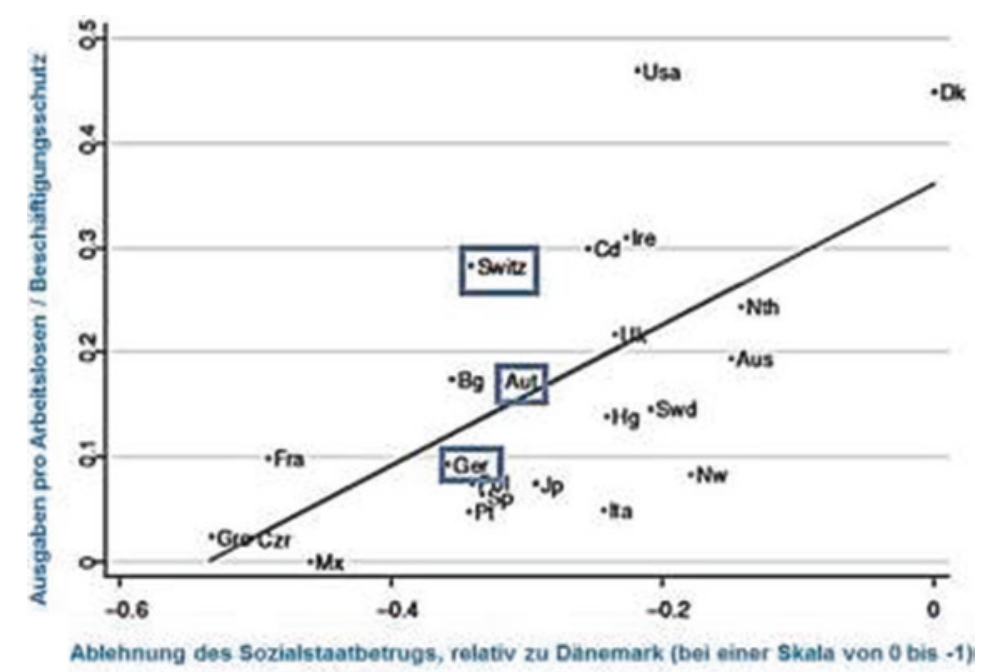

Abb. 1 Korrelation zwischen dem Grad an Gemeinsinn und den Arbeitsmarktinstitutionen. (Quelle: Algan und Cahuc 2009, S. 124)

Es ist zu sehen, dass sich in nordischen Staaten, deren Bürger ein stärkeres Bewusstsein für die Ehrlichkeit gegenüber dem Sozialstaat besitzen, auch ein stärker an Zahlungen orientiertes System etabliert hat. Dementsprechend befinden sich diese Staaten in der Abbildung eher auf der oberen, rechten Seite. Österreich, Deutschland und die Schweiz unterscheiden sich bezüglich der Einstellung nicht allzu sehr, jedoch gibt es beträchtliche Unterschiede in der Ausgestaltung der Arbeitsmarktinstitutionen im Hinblick auf das Verhältnis zwischen Geldleistungen und Kündigungsschutz. Es wird hier mit Durchschnittswerten über alle Erhebungsjahre gerechnet, aber das sich ergebende Muster ist auch im Zeitverlauf konsistent: Änderungen bei der Ausgestaltung des Arbeitslosenschutzes zwischen 1980 und 2000 können zu $78 \%$ mit Änderungen bei der Einstellung der Befragten, also dem Gemeinsinn, assoziiert werden.

Zur Kontrolle werten die Ökonomen noch eine weitere Frage aus: „Würden Sie im Allgemeinen sagen, dass den meisten Menschen vertraut werden kann, oder sollte man eher vorsichtig im Umgang mit Anderen sein?" Damit wird berücksichtigt, dass die Bereitschaft der Bürger, staatliche Sozialversicherungen zu unterstützen, auch davon abhängt, wieviel Vertrauen sie ihren Mitbürgern entgegenbringen. Die Gruppierung nach Ländern spiegelt die vorherigen Ergebnisse: 
Befragte aus den nordisch-skandinavischen Staaten zeigen sich eher vertrauensvoll, während man in kontinentaleuropäischen Ländern und in den Mittelmeerstaaten skeptischer gegenüber seinen Landsleuten ist und bei diesen eine eher höhere Bereitschaft zum Sozialbetrug vermutet.

Die Ergebnisse zeigen eher einen statistischen Zusammenhang als einen wirklichen kausalen Effekt. Zur Überprüfung ihrer These nutzen die Forscher einen besonderen Umstand. Sie können zeigen, dass Amerikaner mit fremden ethnischen Wurzeln vorwiegend die Einstellung ihres Herkunftslandes mitnehmen, auch wenn sie innerhalb der USA geboren wurden. Handelt es sich zum Beispiel bei den Vorfahren eines amerikanischen Befragten um Mexikaner, so ist die Wahrscheinlichkeit, einen Sozialstaatsbetrug inakzeptabel zu finden, um $29 \%$ niedriger als bei einem Landsmann mit dänischen Vorfahren. Bei griechischer Abstammung ist die Wahrscheinlichkeit um $21 \%$ geringer.

Hätten Franzosen, Italiener und Griechen zwischen 1980 und 2000 die gleiche Zivilmoral und damit die gleiche Einstellung gegenüber dem Sozialstaat geerbt wie die Dänen, so hätten laut Studie die Arbeitslosenzahlungen in Frankreich um 8.6 \% höher, in Italien um 9.8 \% und im Fall von Griechenland sogar um $13.6 \%$ höher ausfallen können, und entsprechend weniger hätten diese Länder auf den Kündigungsschutz setzen müssen. Es sei jedoch angemerkt, dass die Studie schon im Jahr 2009 erschien, also bevor die Weltwirtschaftskrise eine Staatenkrise hervorrief und $\mathrm{zu}$ massiven Einschnitten in die Arbeitslosenversicherungen der betroffenen Länder zwang.

Hätten Franzosen, Italiener und Griechen die gleiche Einstellung gegenüber dem Sozialstaat geerbt wie die Dänen, so hätten die Zahlungen an Arbeitslose in Frankreich um $8.6 \%$, in Italien um $9.8 \%$ und in Griechenland sogar um $13.6 \%$ höher ausfallen können.

Die Studie zeigt, dass der Einfluss kultureller Werte auf die Ausgestaltung des Wohlfahrtsstaates nicht unterschätzt werden darf. Zivile Tugenden und ein hoher Gemeinsinn in der Bevölkerung erleichtern ein korrektes Verhältnis zum Staat und ermöglichen bessere Politiklösungen, nicht nur für die Ausgestaltung des Sozialstaates, sondern auch für die Finanzierung anderer Aufgaben z. B. Dank hoher Steuermoral. 
Open Access Dieses Kapitel wird unter der Creative Commons Namensnennung 4.0 International Lizenz (http://creativecommons.org/licenses/by/4.0/deed.de) veröffentlicht, welche die Nutzung, Vervielfältigung, Bearbeitung, Verbreitung und Wiedergabe in jeglichem Medium und Format erlaubt, sofern Sie den/die ursprünglichen Autor(en) und die Quelle ordnungsgemäß nennen, einen Link zur Creative Commons Lizenz beifügen und angeben, ob Änderungen vorgenommen wurden.

Die in diesem Kapitel enthaltenen Bilder und sonstiges Drittmaterial unterliegen ebenfalls der genannten Creative Commons Lizenz, sofern sich aus der Abbildungslegende nichts anderes ergibt. Sofern das betreffende Material nicht unter der genannten Creative Commons Lizenz steht und die betreffende Handlung nicht nach gesetzlichen Vorschriften erlaubt ist, ist für die oben aufgeführten Weiterverwendungen des Materials die Einwilligung des jeweiligen Rechteinhabers einzuholen.

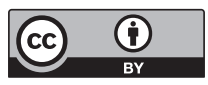

